O.N.R. Research Contract Nonr-222(37), Dept. of Mathematics, University of California at Berkeley, 1957.

4. N. Dunford and J. T. Schwartz, Linear operators, vol. 1, Chapter 5, to appear.

5. W. H. Gottschalk and G. A. Hedlund, Topological dynamics, Amer. Math. Soc. Colloquium Publications, New York, 1955.

6. G. A. Hedlund, A class of transformations of the plane, Proc. Cambridge Philos. Soc. vol. 51 (1955) pp. 554-564.

7. S. Kakutani, On the continuity of eigenfunctions of minimal dynamical systems, to appear.

8. K. Yosida and S. Kakutani, Operator theoretical treatment of Markoff's process and mean ergodic theorem, Ann. of Math. vol. 42 (1941) pp. 188-228.

YALE UNIVERSITY

\title{
ON SPACES WHICH ARE NOT OF COUNTABLE CHARACTER
}

J. M. MARR

It is well known that the unit interval $I$ has a countable base and the fixed point property. By considering the maps $g(x)=x^{2}$ and $h(x)$ $=1-x$, one sees that there is no $x \in I$ such that for every continuous map $f: I \rightarrow I, x \in f(I)$ implies $f(x)=x$.

In Theorem 1 , it is shown that if $A$ is a closed, non-null proper subset of a locally connected, compact Hausdorff space $X$ which has a countable base, then there exists a continuous map $f: X \rightarrow X$ such that $A \cap f(X)$ is not contained in $A \cap f(A)$. Theorem 2 shows that certain nondegenerate topological spaces $X$ contain proper subsets $M$ such that for every continuous map $f: X \rightarrow X, M \cap f(X) \subset M \cap f(M)$. That is, for each of these spaces $X$ and every continuous map $f: X \rightarrow X, x \in M \cap f(X)$ implies $f^{-1}(x) \cap M \neq \varnothing$. The corollary is of interest in that, if $X$ satisfies the hypotheses of Theorem 2 and $M$ consists of a single point, then a fixed point of some of the maps $f: X \rightarrow X$ is located.

Theorem 1. Suppose $X$ is a connected, locally connected, compact Hausdorff space which has a countable base. If $A$ is any non-null, closed, proper subset of $X$, then there exists a continuous map $f: X \rightarrow X$ such that $A \cap f(X) \backslash A \cap f(A) \neq \varnothing$.

Proof. Since $X$ is compact Hausdorff and has a countable base, $X$ is metrizable. Hence $X$ is arcwise connected. Let $y \in X \backslash A$. Since

Received by the editors December 16, 1957 and, in revised form, April 3, 1958. 
$X$ is normal, there exists a continuous map $h$ such that $h(x)=0$ for $x \in A, h(y)=1$, and $0 \leqq h(x) \leqq 1$ for each $x \in X$. Since $X$ is arcwise connected, there is an arc $C$ connecting $y$ and $A$. Now $C$ contains a subarc $C_{1}$, such that $y \in C_{1}$ and $C_{1} \cap A$ is a single point $x_{0}$. Then there is a homeomorphism $g$ such that $g([0,1])=C_{1}, g(0)=y$, and $g(1)=x_{0}$. Consider the continuous map $f=g h$. Clearly $f: X \rightarrow X$ and $x_{0} \in A \cap f(X)$. But since $x_{0} \neq y$ and $f(A)=y, x_{0} \notin f(A)$. Hence $x_{0} \notin A \cap f(A)$; and $f=g h$ is the required map.

In the following let $M$ consist of the set of all points $x \in X$ such that if $x$ is a limit point of $\left\{y_{\alpha}\right\}$ where $U y_{\alpha} \subset X \backslash x$, then $\left\{y_{\alpha}\right\}$ contains uncountably many distinct points. It may be noted that $X$ does not satisfy the first axiom of countability at points of $M$.

THEOREM 2. Let $X$ be a connected Hausdorff space which contains a non-null set $M$ such that $M=\bar{M}$, and $M \neq X$. Suppose also that each point of $X \backslash M$ has a countable base. Then for every continuous map $f: X \rightarrow X, M \cap f(X) \subset M \cap f(M)$.

Proof. Let $f$ be a continuous function such that $f$ maps $X$ into $X$. If $M \cap f(X)=\varnothing$, then $M \cap f(X) \subset M \cap f(M)$. On the other hand, suppose $x \in M \cap f(X)$ and $x \notin f(M)$. Now $x$ is a limit point of $X \backslash x$, for otherwise $X$ would not be connected. Since $x \notin f(M), f^{-1}(x) \subset X \backslash M$. Suppose there exists $z \in f^{-1}(x)$ such that every neighborhood of $z$ intersects $X \backslash f^{-1}(x)$. Since $X \backslash M$ is open and $z \in X \backslash M$, there exists a countable set $\left\{U_{n}(z)\right\}$ of neighborhoods of $z$ such that $\bigcap_{n=1}^{\infty} U_{n}(z)=z$, and $U_{n}(z) \subset X \backslash M$ for each $n$. In each $U_{n}(z)$ there exists a point $u_{n}$ such that $u_{n} \in X \backslash f^{-1}(x)$. Now $f\left(u_{n}\right) \subset X \backslash x$ for each $n$; and, by the continuity of $f, x$ is a limit point of the set $\bigcup_{n=1}^{\infty} f\left(u_{n}\right)$. But $\bigcup_{n=1}^{\infty} f\left(u_{n}\right)$ does not contain uncountably many distinct points. Thus a contradiction has been reached. Suppose that for every $z \in f^{-1}(x)$, there exists a neighborhood $U(z)$ such that $U(z) \cap\left\{X \backslash f^{-1}(x)\right\}=\varnothing$. Clearly $U(z)$ may be taken so that $U(z) \subset X \backslash M$. Then $U(z) \subset f^{-1}(x)$ for every $z \in f^{-1}(x)$, and $f^{-1}(x)$ is open in $X$. Since $x$ is closed in $X, f^{-1}(x)$ is closed in $X$. Therefore $f^{-1}(x)=X$ and $f(X)=x$. But $f(M) \subset f(X)$; hence, $f(M)=x$. But this contradicts the assumption that $x \notin f(M)$.

CoROLlary. If $X$ is a nondegenerate connected Hausdorff space in which $M$ is a single point $x_{0}$, then for every continuous function $f$ such that $f: X \rightarrow X$ and $x_{0} \in f(X), f\left(x_{0}\right)=x_{0}$.

Proof. By Theorem 2, $x_{0} \subset f\left(x_{0}\right)$. Since $f\left(x_{0}\right)$ is a single point, $x_{0}=f\left(x_{0}\right)$.

Kansas State College 